\title{
Reusing Old Computers - An Environment Friendly Method to Produce Computational Performance
}

\author{
Suprakash S, Balakannan S P
}

\begin{abstract}
Disposing the old computer parts to the environment, the toxic chemicals from the hardware mix-up with soil and makes the solid water poisonous. Most of the computers which are made up of a mix of toxic chemicals like lead, mercury, cadmium and similar materials are very much harmful to the environment when they are thrown to the environment without proper disposal. With the rapidly growing number of PCs and different electronics regularly filling landfill regions, any chance to drag out the lifecycle of these components ought to be taken. This paper aims at using such old computing parts which are usable for computations. Also, instead of using heavy PC on the client side, a replacement of thin PC and then client PC will reduce the hardware elements also the power consumption of these machine. Also there is a high demand of computing power in research projects which takes hours of time to produce results. A low scale compute cluster made upon this reusable computer serves this need also. This compute clusters are provided as a virtual server over a private cloud.
\end{abstract}

Keywords: Green Computing, Cluster, PC-Reuse, Virtual cluster.

\section{INTRODUCTION}

$\mathrm{W}$ ith the advancement in the computer hardware and software technologies, most of the hardware a decade old is almost dead in supporting the current software. Home users and industries are moving towards adapting the new technology throwing away the old computers. When this hardware is moved towards landfills, toxic materials in the hardware mixes with soil and water which will in turn gives great imbalance in the human physical system.

There are a lot of initiatives taken around the world in minimization of e-waste and making the computing platform greener. As discussed in [1], Energy Star Program by US-EPA in 1992 made standards on greenhouse gas emission, a major initiative towards environment protection. Followed by the major contributors Dell's Tree Planting program and HP's return and recycling program where HP takes the used computer parts, separates the plastic, metals

Revised Manuscript Received on December 05, 2019

* Correspondence Author

Suprakash S, Department of Information Technology, Kalasalingam Academy of Research and Education, Anand Nagar, Krishnankoil, India. Email: s.suprakash@gmail.com

Balakannan S P*, Department of Information Technology, Kalasalingam Academy of Research and Education, Anand Nagar, Krishnankoil, India. Email: balakannansp@gmail.com and carefully sends for recycling. Where ever possible these materials are also used for new product development. [2][3], the Greenpeace Laboratory published a report during the year 2007 about the usage of hazardous materials within top selling laptop brands. It is clearly reported that these laptops are made with hazardous materials in a greater extent and once it is mixed with environment, it not only harms the environment but also humans and animals. [4][14][15] Reviews deeply an eco-friendly IT from the manufacturing stage to disposal of computer and its parts. It points out the reuse of computer and its parts wherever possible if they are in working conditions.

The three main categories of disposition of old computer hardware are reducing, recycling and reusing. Recycling and reusing has become the solution to reduce the e-waste since computer hardware contains toxic materials [5]. The concept of outdated computers is typically defined by the corporate refresh cycle of 3 to 5 years. As the computers are cycled out of use, they are subject to re-purposing, recycling, or disposal. As more and more computer equipment is being cycled out of use and slated for possible disposal, it is growing more important to find alternative ways to use them.

This work aims at reusing the outdated computers by upgrading them to the minimal requirement. Most of the educational institutions and industries who owns hundreds of computers a decade old is out of date and requires some proper mechanism to reuse or recycle it. This work examines the conditions and possibilities to reuse such PCs. Also since there is growing requirement of high performance and parallel systems from the research, these systems are clustered to form small scale high performance computers.

Cluster [6] is formed by combining many different outdated PCs, which have enough computing power to execute the task in turn reduces the e-waste and have less harmful impact on environment. The main objective is to form cluster with outdated PCs with minimal modification to get high computing power for execution.

The rest of the paper is structured as follows. Section-2 describes about the related works. Section-3 explains the methodology of the work. Section-4 explains the setup and analysis of the proposed work. 


\section{RELATED WORKS}

[7] Super Computers from Clusters used more than 100 PCs to provide supercomputing power. They worked on with the configurations available without any modifications on the hardware. These systems worked parallel to get the jobs done. This cluster spotted the eco region on the world map with different colors. [8] VITERAAS, a virtual cluster created with three groups of clusters. First with normal working PCs, second with Servers and the third with Amazon HPC. Here too parallel jobs from the University is run on this cluster groups and whenever there is a scale-up amazon services are used.

[9] Created a virtual cluster VC3, similar to the services offered by amazon and Google. The problem addressed was to create multi institution collaboration without going for high-end computing devices. They used the systems available with them to create the cluster environment.

Carlos et.al [10] described an architecture which was designed to make simpler the creation of all necessary computing platforms and flexible development of the IaaS. The architecture aims to scale in and out the infrastructure when needed. Marcos et.al [11] investigated the benefits of scheduling strategies and evaluated the cost by considering the use of the resources from the cloud and found naïve scheduling strategies had a significant impact of using remote resources.

Ruben et.al [12] presented a generic cluster architecture, that offers cluster consolidation, partitioning of cluster and support for the heterogeneous environment. Also, they have proposed a model to characterize the cluster using cloud resources. The architecture was evaluated in the execution of high throughput computing workloads and it has proven that the cluster comprised of a workable and carried out in HTC platform. Feifie et.al [8] proposed a novel elastic resources allocation strategy named as Mixed Bin Packing Algorithm, which aims to improve the system efficiency by adjusting the resources at run time and minimize the cost of users.

Miguel et.al [13] came up with a tool called Elastic Cloud Computing Cluster that design virtual cluster over the IaaS cloud using green computing technology. Depends on the user needs, cluster scale in and scale out the resources.EC3 employed with an energy manager system, CLUES that provide the cluster to adapt elastic facilities. Paul et.al [10] presented architecture with extended an open source elastic resource manager with light weight REST-based broker to transmit the information of host between nodes in context.

\section{METHODOLOGY}

A cluster is defined as a group of loosely coupled systems operating together as a single system to achieve high performance computing. Here cluster is created with old outdated PCs. The architectural model to setup the system is shown in the figure 1 . The required and necessary components, the motherboard and hard discs are separated out and cluster is created. The rest of the materials are taken for recycling or safe disposal.

\section{A. Cluster formation}

Here cluster computer is a grouping of outdated computers connected together by fast local network through a network switch to make the cluster perform as a single entity. The access point of the cluster is a preferably high end computer connected with two Ethernet ports one for the cluster group and the other for the public network access. So there is no additional cost other than network setup and power supply to the cluster nodes.

The challenge on the system was to dispose the separated parts in a proper way. But thanks to HCL and similar companies who takes care of the e-waste disposal in proper way.

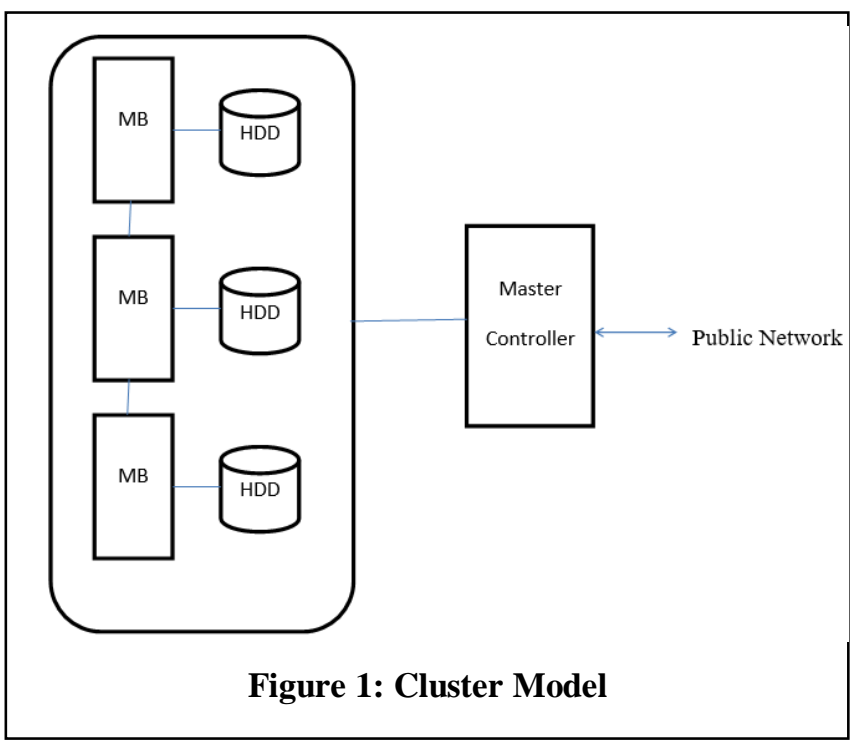

\section{B. Configuration setup}

After making all the connection between the frontend, nodes, switch, and the Internet. Next step is to check before installation software is that eth0 on the frontend must be connected to the Ethernet switch and eth 1 must be connected to the external public network. Each node collects metric data on itself and distributes the information whenever an update occurs. All nodes listen for these updates; therefore, all nodes maintain data on the status of the entire cluster. Figure 2 shows the frontend and compute node setup.

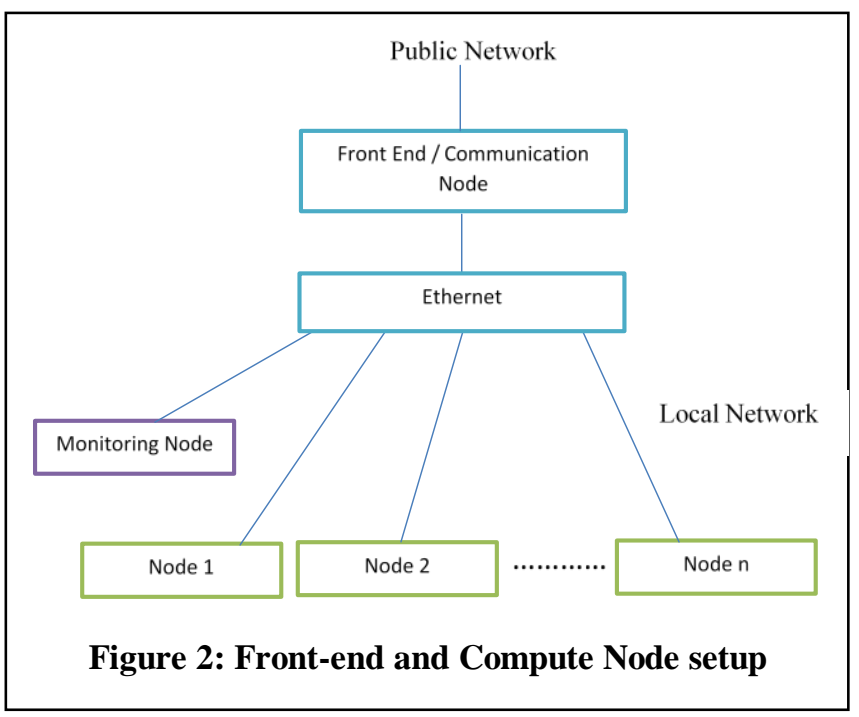




\section{IP Setup}

In the case, if the IP for the cluster was set up as static IP. There is no DHCP server on the network to answer the DHCP setup request from the frontend and the user will have to supply the information. This is a small service running in background that collects the metric data from the machine it is on and uses the listen/announce protocol to transmit this data over TCP.

\section{Application Setup}

The cluster formed can be used in different applications. Most of the clusters are designed for high availability and high performance computing. The cluster formed here is utilized as a load balancing web cluster. The key benefits that load balanced web servers will provide are,

Scalability: The amount of traffic a web site receives includes a substantial impact on its performance, and load balancing provides the potential to handle most unexpected spikes in traffic by spreading the traffic across multiple servers. Adding a lot of load balanced servers to handle exaggerated traffic is way easier and quicker to implement than moving a web site to a completely new, a lot of powerful server. This is often particularly advantageous for sites that operate virtual web servers, since existing servers will simply be cloned and supplemental to the load balanced array.

As a site's traffic fluctuates, load balancing permits server administrators to extend or decrease the amount of web servers counting on the site's current requirements. Here are a couple of samples of however regular changes in web site traffic may necessitate the utilization of load balancing to regulate server capacity:

Educational web sites: Universities that use their website to permit students to register for courses on-line can typically see an oversized increase in site traffic every semester throughout the enrollment time. This increase will cause web site slowness at these peak times, and adding one or a lot of load balanced web servers to extend the site's capability will address this issue.

E-commerce sites: E-commerce sites typically see an oversized increase in traffic over the vacation season; adding one or a lot of load balanced web servers will keep the location from experiencing slowness throughout this busy time. Changes to the amount of load balanced servers will be created as required, thus servers will be supplemental in after they aren't any longer necessary. Utilizing load balancing provides this exceptional measurability, ensuring that a web site is often ready to satisfy its users' demands

Redundancy: Utilizing load balancing to take care of internet services on multiple one web server (whether the servers can be dedicated servers or virtual servers) will greatly limit the impact of hardware failure on a site's overall uptime. Since the web traffic is shipped to multiple or additional web servers, if one server fails, the load balancer can immediately transfer the traffic to the next operating web server(s). preparation for periods of exaggerated traffic, then removed

Flexibility: Using multiple load balanced servers to handle a web site's traffic permits administrators to perform maintenance on a server while not impacting site uptime. This could be done by routing all traffic specific server and putting the load balancer in active/passive mode. Package upgrades and code updates may be deployed to the passive server and tested in the production atmosphere, and once administrators are done with the updates, they will switch the passive server to active and do identical work on the rest of the server(s). Any server maintenance may be staggered during this approach, with a minimum of one server remaining accessible, guaranteeing that the site's users do not feel any outages.

The setup uses nginx plus as a web server and load balancer. The same setup is replicated with all cluster nodes. The load balancer module is configured with cluster nodes and uses default round-robin method for load sharing. The usual setup takes all the clusters in the live mode and all systems will be always active leaving a high power footage all the time. But whenever the requests are less and handled by one or two clusters the other cluster nodes can be put to the sleep mode where RAM alone is active and other parts are powered off, saving power.

The figure 2 shows the cluster management model designed for the web clusters to put systems on sleep mode. Worker_processes are set to the number of cpu cores available. Worker connections are the number of concurrent user connections to the cluster. This vale is set to 1024 (512 connections each request from bowser takes two active connections). $\mathrm{MCi}$ is the maximum number of connections to the client and $\mathrm{Ci}$ refer to ith cluster.

\section{RESULTS AND ANALYSIS}

This section gives overview of the software requirements and installation necessary to setup a productive cluster capable of taking workloads from the research departments. As mentioned, Rocks cluster distribution is used for cluster creation. For the front end or called as the the master node is setup with 2 GB RAM and 40 GB HDD. Two physical Ethernet ports are added to the machine, one for the cluster access and the other for the public network. For the cluster nodes 28 systems are taken and found 20 systems in reusable condition. These systems are setup with 2 GB RAM and 40 GB HDD and a single Ethernet port. For all these 20 systems, only the required components are separated out and integrated with cluster. The other systems components like CPU cabinet, CD/DVD drive, additional fan are taken out and send for safe disposal. Table 1 describes the configurations for the setup.

As discussed in the section III, the cluster setup is made for web server for running University application. Nginx plus is installed in all the cluster nodes serving as a web server. Also Nginx is used as the load balancer. The controller node is added to the cluster with the setup described in the figure 2 . 
Reusing Old Computers - An Environment Friendly Method to Produce Computational Performance

Table 1: Cluster Hardware Requirements

\begin{tabular}{|l|l|}
\hline Frontend Node & \\
\hline Hard Disk & Minimum 30GB \\
\hline Memory Capacity & $2 \mathrm{~GB}$ \\
\hline Ethernet & $\begin{array}{l}\text { 2 Physical ports } \\
\text { One for cluster communication } \\
\text { and other for public network }\end{array}$ \\
\hline Compute Node & $30 \mathrm{~GB}$ \\
\hline Hard Disk & $2 \mathrm{~GB}$ \\
\hline Memory Capacity & 1 Physical port \\
\hline Ethernet &
\end{tabular}

A sample set of web request are manually generated with static page content and submitted to the load balancer for testing. The results of the same is depicted in Figure 3 and Figure 4. Out of the 20 nodes selected, 2 nodes are always on to provide basic services. Other nodes are made to sleep state by the controller. When the number of incoming user connections increases, the controller wakes up the next sleeping node and put the status of the node as active in to the upstream servers of nginx.

Considering the power measurements, it is found that an average of 124 Watts is consumed by the machine without considering the monitor power. Figure 5 shows the power rating of the systems. From the machines, the CPU cabinet, CD/DVD, additional FANs are taken out for which an average power consumption of 18 Watts is getting reduced for each machine. That is an individual node is consuming about 116 Watts on an average. These systems on the sleep mode is powering up the RAM alone and power to other parts are cutoff. This is found to be 3 Watts per machine on an average. As shown in the Figure 4, the overall power consumption of the entire system can be reduced to greater extent with respect to the load on the system. When there is a heavy load the power consumption is high but reduces to a greater extend when there is a less load.

\section{CONCLUSION}

Hundreds of computing systems which are outdated are taken in to consideration for reusing the systems in a proper way without throwing away or dumping in to landfills. Clustering these systems is giving the performance of a low scale high performance computer. Power considerations are made and a renewable energy technology is considered to power-up the systems. The work considered the high frequency of the university web servers during the peak hours of course registration, online booking systems. Load balancing server can equally distribute the incoming load and the controller designed takes care of the machines power states. Utilizing these technology can reduce the old systems from throwing to landfills and at the same time providing a good service for the web applications. Few cases are taken from the research departments of Biotechnology and chemical which requires a parallel computer or a high performance computer to complete the tasks. This will be taken as a future work and the performance of the systems will be evaluated.
Activate Client Nodes

Add Client configurations(worker_processes, worker_connections, status_of_client) to configuration Module

Set MAX Connections to each Clients-MCi

Set Primary Server CO

for each cluster

ADD Servers to load balancer:

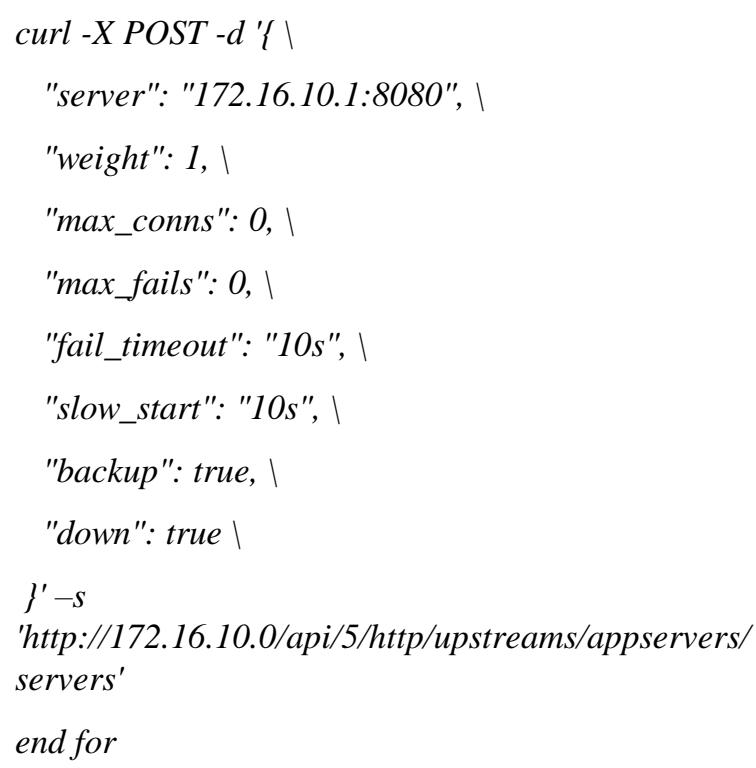

Monitor Connections:

for each active clusters $\mathrm{Ci}$

GET active connections $C i$ active

Check for Connection Limits

C_Limit $=C i-C i$ active

if $\left(C_{-}\right.$Limit $\left.>=M C i\right)$

Activate New Cluster from Deactivated Cluster Pool

curl -X PATCH -d '\{ "down": false\}' -s

'http://172.16.10.x/api/5/http/upstreams/appservers/ser vers $/ P i^{\prime}$

if $($ Ci_Active $<=0)$

Deactivate the connection and put to sleep mode

curl -X PATCH -d '\{ "down": true \}' -s

'http://172.16.10.x/api/5/http/upstreams/appservers/ser vers $/ P i^{\prime}$

Figure 2: Cluster Management 
No. of Clusters Used

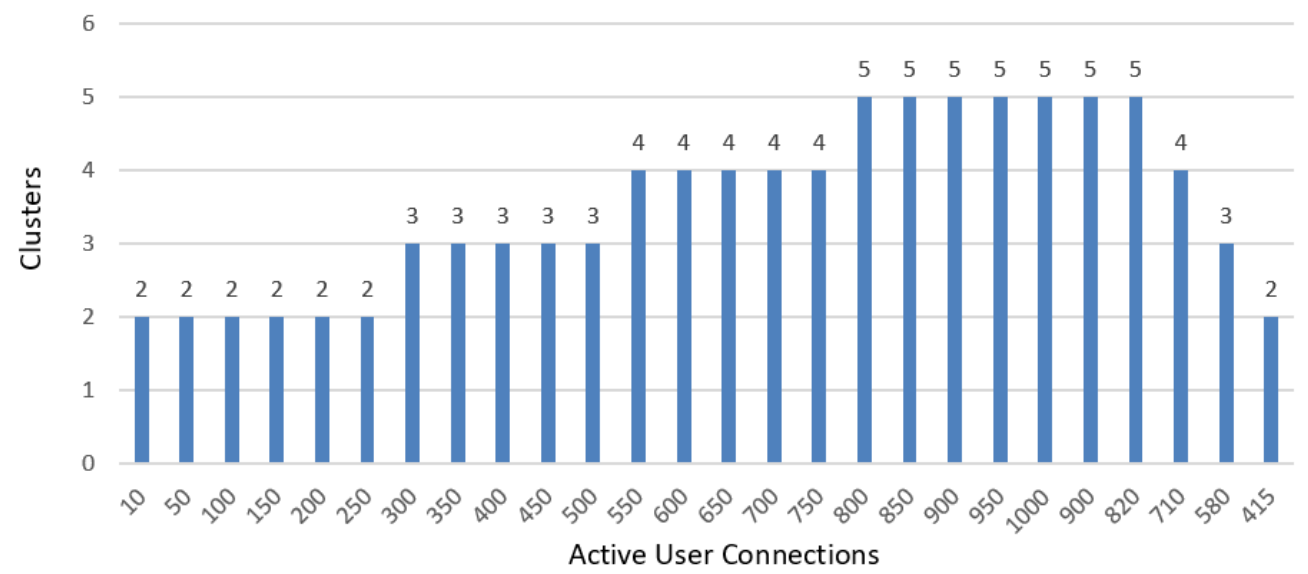

Figure 3: Cluster Nodes user with respect to different user connections

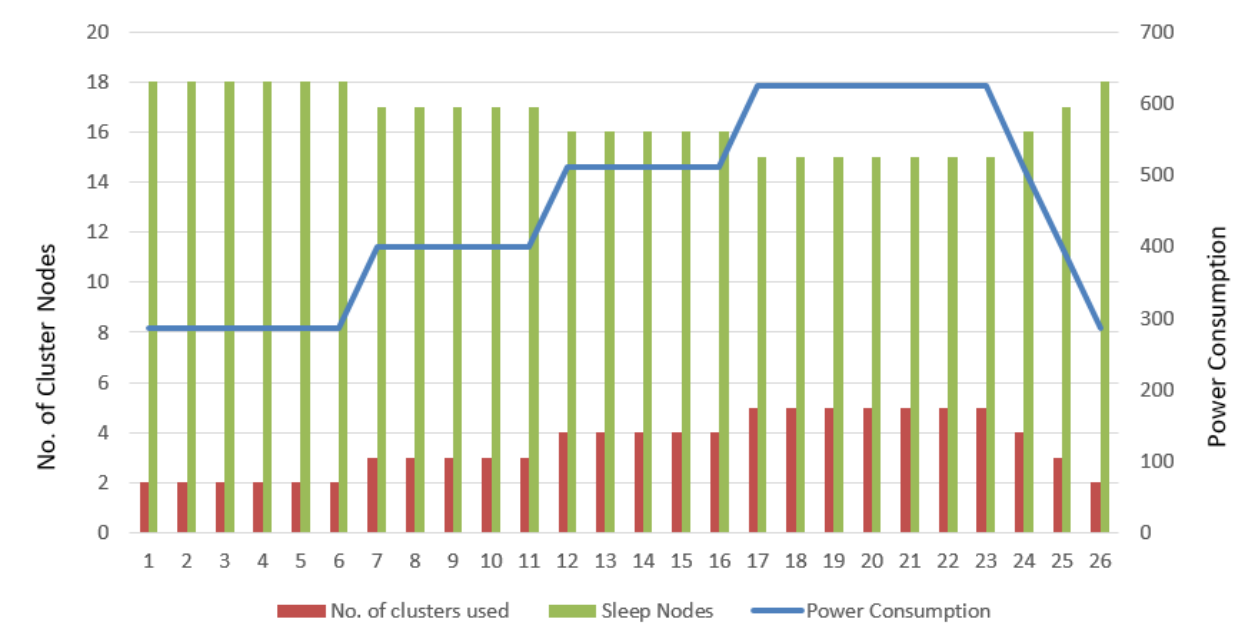

Figure 4: Power consumption

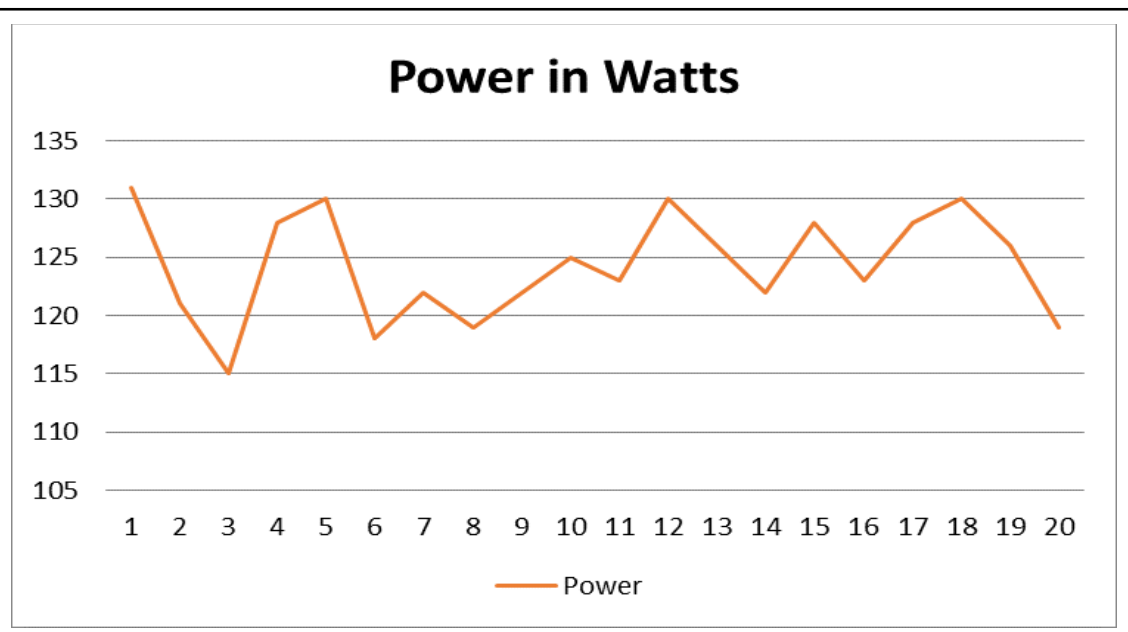

Figure 5: Power Consumption before disassembling 


\section{REFERENCES}

1. Mesaad, Mariam, et al. "Survey on the Global Green Computing Initiatives." International Journal of Computer Applications 167.7 (2017)

2. Brigden, K. \&Santillo, D. (2007): Analysis of hazardous substances in a HCL laptop computer; Greenpeace Research Laboratories Technical note $02 / 07$

3. Brigden, K., Webster, J., Labunska, I. \&Santillo, D. (2007): Toxic chemicals in computers reloaded; Greenpeace Research Laboratories Technical note $06 / 07$

4. Raza et al. T "A Review on Green Computing for Eco-Friendly and Sustainable IT" J. Comput. Intell.Electron. Syst. 2012, Vol. 1, No. 1, doi:10.1166/jcies.2012.1023

5. $\quad$ R. Panda, "E-waste Management: A Step towards Green Computing," International Journal of Environmental Engineering and Management., ISSN 2231-1319, Volume 4, Number 5 (2013), pp. 417-424

6. Yeo CS, Buyya R, Pourreza H, RasitEskicioglu M, Graham P, Pourreza P, Sommers F (2006) Cluster computing: high-performance, high-availability, and high-throughput processing on a network of computers. In: Zomaya AY (ed) Handbook of nature-inspired and innovative computing. Springer, Berlin, pp 521-551

7. Hargrove, William W., Forrest M. Hoffman, and Thomas Sterling. "The Do-It-Yourself Supercomputer." Scientific American 285, no. 2 (2001):72-79. doi:10.1038/ SCIENTIFICAMERICAN 0801 - 72

8. F. Doelitzscher, M. Held, C. Reich and A. Sulistio, "ViteraaS: Virtual Cluster as a Service," 2011 IEEE Third International Conference on Cloud Computing Technology and Science, Athens, 2011, pp. 652-657. doi: 10.1109/CloudCom.2011.101

9. Lincoln Bryant, Jeremy Van, Benedikt Riedel, Robert W. Gardner, Jose Caballero Bejar, John Hover, Ben Tovar, KenyiHurtado, and Douglas Thain. 2018. VC3: A Virtual Cluster Service for Community Computation. In Proceedings of the Practice and Experience on Advanced Research Computing(PEARC '18). ACM, New York, NY, USA, Article 30, 8 pages. https://doi.org/10.1145/3219104.3219125

10. Carlos de Alfonso, Miguel Caballer, Fernando Alvarruiz, GermánMoltó and Vicente Hernández , "Infrastructure deployment over the cloud", IEEE, 2011.

11. Marcos Dias de Assuncao, Alexandre di Costanzo, RajkumarBuyya, "A Cost- benefit analysis of using cloud computing to extend the capacity of clusters", Cluster Comput ,13: 335-347,2010.

12. Ruben.S Montero, Rafael Moreno-Vozmediano, IgancioM.Llorente, "An elasticity model for Hight Throughput Computing clusters", J.ParallelDistrib. Comput., 2011.

13. Feifei Liu, XiaosheDong,"'A Novel elastic Resource Allocation Strategy of Virtual cluster", IEEE,2011

14. Karuppasamy, M., Suprakash, S., Balakannan, S.P.,"Energy efficient utilization of cloud resources using hybrid ant colony genetic algorithm for a sustainable green cloud environment ", Proceedings of 2017 International Conference on Intelligent Computing and Control, I2C2 2017 .

15. Karuppasamy, M., Balakannan, S.P. "Energy saving from cloud resources for a sustainable green cloud computing environment",Journal of Cyber Security and Mobility,2018

\section{AUTHORS PROFILE}

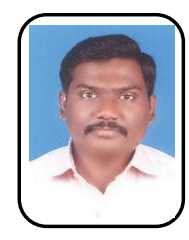

S Suprakash received his Bachelor degree in Computer Science and Engineering from Anna University by 2006. He has received his Master degree in VLSI Design from Anna University, 2008. He is working as an Assistant Professor in the Department of Information Technology, Kalasalingam Academy of Research and Education and also doing his research under the area Green Cloud Computing. He is the technical lead and undergoing many projects in reducing paper waste and automation. His areas of interest are Computing, Security, Data Management and Networks.

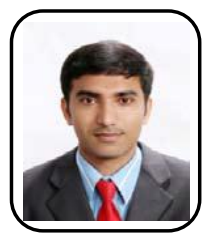

Balakannan S.P received his Ph.D. degree from the Department of Electronics and Information Engineering at Chonbuk National University, South Korea (2010). He has received his master degree (5 years integrated) from the Department of Computer Science and Engineering, Bharathiar University, India, in the year 2003. He has worked as a Project Assistant in Indian Institute of Technology (IIT), Kharagpur, India from 2003 to 2006. Currently, he is working as Assistant Professor in the Department of
Information Technology, Kalasalingam Academy of Research and Education, Tamilnadu, India. His areas of interest include Wireless Network, Network Coding, Cloud \& Green Computing, Cryptography, and Mobile Communication. 